\title{
Unusual Cause for Massive Pediatric Lower Gastrointestinal Bleed with Atypical Postoperative Intestinal Sequelae
}

\author{
Suhasini Gazula* \\ Department of Pediatrics Surgery, ESIC Super speciality Hospital, Sanathnagar, Hyderabad, India
}

Received: November 28, 2015; Accepted: January 20, 2016; Published: January 30, 2016

*Corresponding author: Suhasini Gazula, Super Specialist - Senior Scale \& Head, Department of Paediatric Surgery, ESIC Super speciality Hospital, Sanathnagar, Hyderabad, India, E-Mail: suhasinigazula@gmail.com

\section{Introduction}

Nearly $25 \%$ of the global population is infested with Ascaris lumbricoides [1]. While persons of any age group can be infested with ascaris, children between the ages of 4 and 14 years most commonly harbor the parasite [2]. Chronic infestation with Ascaris lumbricoides (roundworm), leading to malnutrition, microscopic blood loss and anemia are common among children in developing countries. It also presents with acute complications like intestinal obstruction, perforation and biliary obstruction $[3,4]$. Volvulus and bowel gangrene, the two most serious intestinal complications, are less frequent and massive gastrointestinal (GI) bleeding has been only sporadically reported [5-10]. Severe necrotizing bowel or biliary inflammation caused by secretory toxins and decomposition products of roundworms has also been rarely reported [11,12].

We report a 2-year old child who presented with massive lower GI bleed as the presenting feature due to volvulus and gangrene of the ileum caused by roundworm bolus. He also developed early extensive bowel adhesions which have been seldom reported following ascariasis and can be probably attributed to worm toxins. Though primary anastomosis after resection of a bowel segment with a worm bolus may be the generally acceptable procedure, in cases with evidence of serosal contact with worm toxins is present, a bowel diversion procedure may be a better option. Mechanisms for intestinal obstruction and intestinal sequelae in ascariasis are also discussed.

\section{Case Report}

A 2-year old male child presented to the emergency room with two day history of generalized pain abdomen and profuse bleeding per rectum. There was no history of abdominal distension, bilious vomiting or hematemesis. Clinical examination revealed a critically ill child with tachycardia (Pulse 110/min), mean BP 70mm Hg and severe pallor. On abdominal examination, there was mild abdominal distension and severe generalised guarding which precluded further clinical examination. On digital rectal examination, he passed copious amounts of altered blood. Significant laboratory investigations were hemoglobin of $4 \mathrm{gm} / \mathrm{dL}$ and leucocytosis with neutrophilia. Plain erect X-ray of the abdomen showed multiple air-fluid levels. Ultrasonography findings were obscured by excessive bowel gas and demonstrated dilated bowel loops and probable volvulus of the small bowel. The child was adequately resuscitated with intravenous fluids, antibiotics and red cell transfusion. As the child was critically ill, no further imaging was done. Once stabilized, an exploratory laparotomy was performed which revealed the jejunum and ileum filled with 150-200 roundworms with volvulus and extensive gangrene of the ileum (Figure 1). The ileum showed areas of impending rupture and seropurulent discharge on its serosal surface. The necrotic bowel containing worms was immediately resected (Figure 2). By milking the proximal bowel, more worms were expressed out and copious amounts of altered blood were

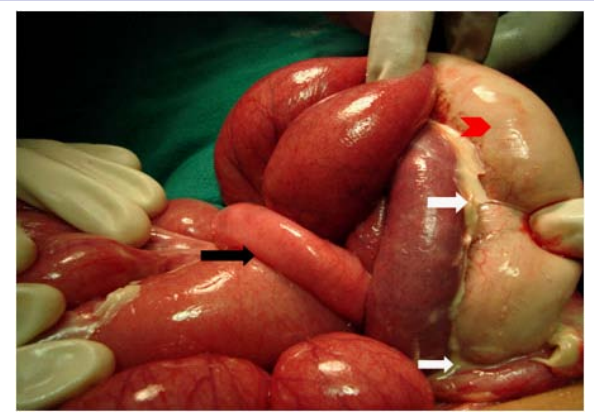

Figure 1: Intraoperative photograph showing volvulus of ileum (black arrow), gangrenous necrotic ileum (grey arrowhead) and seropurulent discharge coating the serosal surface of the small bowel (white arrows).

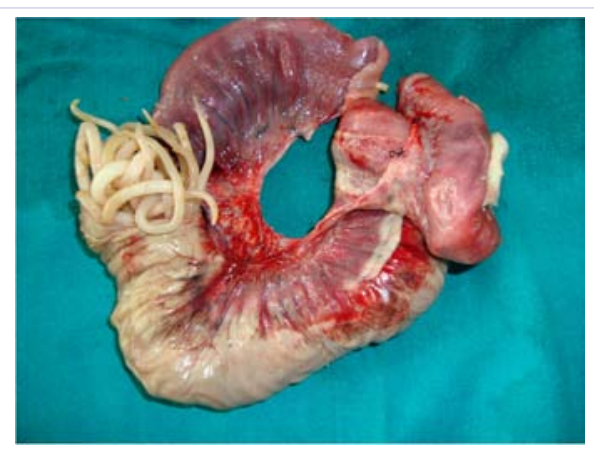

Figure 2: Photograph of the resected ileum specimen teeming with numerous roundworms. 
obtained on decompressing the distal bowel. Peritoneal lavage was done and a double barrel ileostomy was fashioned as a time-saving procedure. Postoperatively, the child was started on anthelminthic piperazine citrate following which 2-3 worms passed out via the ileostomy. Retrospectively, the parents recalled that the child had a history of passing roundworms on and off per rectum. The child had an uneventful recovery and 2 weeks later the ileostomy was closed. However, the child developed adhesive intestinal obstruction within 7 days of the ileostomy closure which did not resolve with conservative management. Hence a redo-laparotomy was done and extensive interloop adhesions of small bowel were lysed. The child recovered well and had no further complications and is well at 12 months follow up.

\section{Discussion}

Ascariasis is a staggering worldwide public health problem and the most common helminthic infestation in the world. Factors like poor sanitation, crowded living conditions and pickling of raw vegetables are implicated in the prevalence of ascaris [13]. Our case is noteworthy in that this child with ascariasis presented with massive lower gastrointestinal bleeding as the chief symptom which is hitherto unreported in children and hence ascariasis was not suspected as a cause initially. In addition, he also developed severe postoperative intestinal sequelae which have been seldom described. Our choice of ileostomy as the primary surgical procedure in a critically ill child instead of the generally accepted resection and anastamosis may have serendipitously helped us avert anastomotic leak and its devastating consequences.

The most frequent clinical presentation in ascariasis is intermittent colicky abdominal pain and incomplete bowel obstruction [11,14]. Heavy infestation results in a tangle of adult worms producing subacute intestinal obstruction. It is imperative to recognize these complications expeditiously and initiate early, aggressive management $[5,12]$. Plain radiographs may reveal a mass of worms described as "whirlpool pattern" in addition to air-fluid levels though this sign was not evident in our patient [15]. The roundworms may be detectable as tubulous, moving, echogenic structures on ultrasonography with anechoic fluid filled alimentary canal of the worm described as "the inner tube sign"[16]. Majority of children with subacute intestinal obstruction resolve spontaneously on conservative treatment with bowel rest, antispasmodics, anthelminthics, and intravenous fluids [14]. Hamid et al reported that while use of gastrograffin resulted in faster relief of signs and symptoms of Ascaris lumbricoides induced subacute bowel obstruction with early passage of worms/flatus and return to oral feeds, its role in reducing the likelihood of laparotomy remained inconclusive [17]. Other acute conditions include hepatobiliary and pancreatic ascariasis, acute appendicitis, bowel perforation, peritoneal granulomas, small-bowel volvulus and intussusceptions [18]. Volvulus and ischaemic bowel, the two most serious intestinal complications, account for less than $10 \%$ of cases [5] but warrant prompts surgical intervention. In our case too, a high index of suspicion and timely surgical intervention helped us manage the child.
The factors responsible for ascaris-related intestinal obstruction include; a) multiple worms forming a large bolus causing mechanical obstruction, which is most common, b) worm bolus may serve as a lead point in intussusception or a pivot in volvulus, c) worms may inhabit the ileocecal valve and secrete neurotoxins which prompt small bowel contraction; which, alongwith high worm burden can obstruct the intestine, d) host inflammatory reaction to worm-derived hemolysins, endocrinolysins, and anaphylatoxins can also be severe enough to obstruct the gut lumen [15].

Our patient also developed early severe postoperative adhesions and by using bowel diversion (ileostomy) as the initial procedure, we were able to avoid ileal anastomotic leak in the early recuperative period. It has been shown that worm secretions and decomposition products of disintegrating worms are capable of provoking a severe reaction in the bowel which may be distant from the site of the primary worm bolus $[11,12]$. Moreover, when the worm enters the peritoneal cavity, the host immune response may result in masses of inflamed tissue and granulomas seeded throughout the greater omentum, serosal covering of the liver and spleen, and the peritoneum [19]. Steinberg et al reported severe postoperative intestinal sequelae in 2 infants with ascaraisis presumably in response to serosal contamination by worm fragments at the initial surgery [12]. Being faced with intestinal obstruction or anastomotic leak in the immediate postoperative period in a critically ill child may have devastating consequences. Our report as well as the aforementioned report illustrate that though primary anastomosis after resection of a bowel segment with a worm bolus may be generally acceptable, in cases where evidence of perforation or serosal contact with the worm toxins is present, a bowel diversion procedure may be a better option, more so in a critically ill child.

In summary, this case highlights the importance of considering ascaris infestation as a cause of lower gastrointestinal bleeding among pediatric patients without hint of other causes and need for prompt surgical intervention in this ominous scenario. Also, one should be aware of possible sequelae and exercise caution while planning the surgical procedure. Surgical procedure should be modified in the presence of suspected bowel wall contact with worm products.

\section{References}

1. Bethony J, Brooker S, Albonico M, Geiger SM, Loukas A, Diemert D, et al. Soil-transmitted helminth infections: ascariasis, trichuriasis, and hookworm. Lancet. 2006;367(9521):1521-1532.

2. Khuroo MS. Ascariasis. Gastroenterol Clin North Am. 1996;25(3):553577.

3. Sangkhathat S, Patrapinyokul S, Wudhisuthimethawee $P$, Chedphaopan J, Mitamun W. Massive Gastrointestinal Bleeding in Infants with Ascariasis. J Pediatr Surg 2003;38(11):1696-1698.

4. Mukhopadhyay B, Saha S, Maiti S, Mitra D, Banerjee TJ, Jha M, et al. Clinical appraisal of Ascaris lumbricoides, with special reference to surgical complications. Pediatr Surg Int 2001;17(5-6):403-405.

5. Rode H, Cullis S, Millar A, B. Cremin, S. Cywes. Abdominal complications 
of Ascaris lumbricoides in children. Pediatr Surg Int. 1990;5(6):397401.

6. Jacob GS, Al Nakib B, Al Ruwaih A. Ascaris producing upper gastrointestinal hemorrhage. Endoscopy. 1983;15(2):67.

7. Bhasin DK, Chhina RS. Hematemesis in gastric ascariasis. Am J Gastroenterol. 1989;84(12):1585-1586.

8. Wilairatana P, Wilairatana S, Charoenlarp P. Gastric ascariasis associated with upper gastrointestinal hemorrhage. Southeast Asain J Trop Med Public Health. 1994;25(2):401.

9. Pontes JM, Leitao MC, Portela F, Andrade P, Lopes H, Vasconcelos H, et al. Gastric ascariasis: A rare cause of upper gastrointestinal bleeding. Endoscopy. 1996;28(9):792-793.

10. Sarda AK, Kannan R. Massive lower gastrointestinal hemorrhage due to ascariasis. Am J Gastroenterol. 1992;87(9):1233.

11. Louw JH. Abdominal complications of Ascaris lumbricoides infestation in children. Br J Surg. 1966;53(6):510-521.

12.Steinberg R, Davies J, Millar AJW, Brown RA, Rode H. Unusual intestinal sequelae after operations for Ascaris lumbricoides. Pediatr Surg Int. 2003;19(1-2):85-87.
13. Murray CL, Lopez AD. Global Health Statistics: A Compendium of Incidence, Prevalence and Mortality Estimates for Over 200 Conditions. Vol II. Boston: Harvard University Press; 1996:394-405.

14. Rode H, Cullis S, Millar A, Abdominal complications of Ascaris lumbricoides in children. Pediatr Surg Int. 1990;5(6):397-401.

15.Vilamizar E, Mendez M, Bonilla, Varon H, de Onatra S. Ascaris lumbricoides infestation as a cause of intestinal obstruction in children: experience with 87 cases. J Pediatr Surg. 1996;31(1):201-205.

16. Lynser D, Handique A, Daniala C, Pranjal P, Evarisalin M. Sonographic images of hepato-pancreatico-biliary and intestinal ascariasis: A pictorial review. Insights Imaging. 2015;6(6):641-646. doi: 10.1007/ s13244-015-0428-7.

17. Hamid R, Bhat N, Baba A, Mufti G4, Khursheed S, Wani SA, Ali I, et al. Use of gastrografin in the management of worm-induced small bowel obstruction in children. Pediatr Surg Int. 2015;31(12):1171-1176. doi: 10.1007/s00383-015-3793-x.

18. Akgun Y. Intestinal obstruction caused by Ascaris lumbricoides. Dis Colon Rectum. 1996;39(10):1159-1163.

19. Ochoa B. Surgical complications of ascariasis. World J Surg.1991;15:222-227. 